\title{
On Suitability of Euclidean Embedding for Host-based Network Coordinate Systems
}

\author{
Sanghwan Lee, Zhi-Li Zhang, Member, IEEE, Sambit Sahu, Member, IEEE, Debanjan Saha, Member, IEEE,
}

\begin{abstract}
In this paper, we investigate the suitability of embedding Internet hosts into a Euclidean space given their pairwise distances (as measured by round-trip time). Using the classical scaling and matrix perturbation theories, we first establish the (sum of the) magnitude of negative eigenvalues of the (doublycentered, squared) distance matrix as a measure of suitability of Euclidean embedding. We then show that the distance matrix among Internet hosts contains negative eigenvalues of large magnitude, implying that embedding the Internet hosts in a Euclidean space would incur relatively large errors. Motivated by earlier studies, we demonstrate that the inaccuracy of Euclidean embedding is caused by a large degree of triangle inequality violation (TIV) in the Internet distances, which leads to negative eigenvalues of large magnitude. Moreover, we show that the TIVs are likely to occur locally, hence the distances among these closeby hosts cannot be estimated accurately using a global Euclidean embedding. In addition, increasing the dimension of embedding does not reduce the embedding errors. Based on these insights, we propose a new hybrid model for embedding the network nodes using only a 2-dimensional Euclidean coordinate system and small error adjustment terms. We show that the accuracy of the proposed embedding technique is as good as, if not better, than that of a 7-dimensional Euclidean embedding.
\end{abstract}

Index Terms-Euclidean Embedding, Triangle Inequality, Suitability

\section{INTRODUCTION}

Estimating distance (e.g., as measured by round-trip time or latency) between two hosts (referred as nodes hereafter) on the Internet in an accurate and scalable manner is crucial to many networked applications, especially to many emerging overlay and peer-to-peer applications. One promising approach is the coordinate (or Euclidean embedding) based network distance estimation because of its simplicity and scalability. The basic idea is to embed the Internet nodes in a Euclidean space with an appropriately chosen dimension based on the pairwise distance matrix. The idea was first proposed by $\mathrm{Ng}$ et al [2]. Their scheme, called GNP (Global Network Positioning), employs the least square multi-dimensional scaling (MDS) technique to construct a low dimensional Euclidean coordinate

An earlier version was published in ACM Sigmetrics/Performance '06 ([1]). This work is supported in part by the research program 2007 of Kookmin University in Korea, the National Science Foundation grants ITR0085824, CNS-0435444, CNS-0626812, and CNS-0626808, and an IBM Faculty Partnership award.

Sanghwan Lee is with School of Computer Science, Kookmin University, Seoul Korea, e-mail: sanghwan@kookmin.ac.kr.

Zhi-Li Zhang is with Department of Computer Science and Engineering, University of Minnesota, twin cities, Minneapolis, MN USA 55455, e-mail: zhzhang@cs.umn.edu.

Sambit Sahu and Debanjan Saha are with IBM T.J. Watson Research Center, Hawthorne, NY 10532, e-mail: \{sambits,dsaha\}@us.ibm.com.

Manuscript received Feb 25, 2008 system, and approximate the network distance between any two nodes by the Euclidean distance between their respective coordinates. To improve the scalability of GNP, [3] and [4] propose more efficient coordinate computation schemes using Principal Component Analysis (PCA). Both schemes are in a sense centralized. Methods for distributed construction of Euclidean coordinate systems have been developed in [5], [6]. In addition, [5] proposes to use height vector to account for the effect of access links, which are common to all the paths from a host to the others.

While most studies have focused on improving the accuracy and usability of the coordinate based distance estimation systems, other have demonstrated the potential limitations of such schemes. For example, [7] shows that the amount of the triangle inequality violations (TIVs) among the Internet hosts are non-negligible and illustrates how the routing policy produces TIVs in the real Internet. They conjecture that TIVs make Euclidean embedding of network distances less accurate. [8] proposes new metrics such as relative rank loss to evaluate the performance and show that such schemes tend to perform poorly under these new metrics. A brief survey of various embedding techniques is found in [8]. In addition, [9] claims that the coordinate based systems are in general inaccurate and incomplete, and therefore proposes a light weight active measurement scheme for finding the closest node and other related applications.

In spite of the aforementioned research on the coordinate based network distance estimation schemes regardless of whether they advocate or question the idea, no attempt has been made to systematically understand the structural properties of Euclidean embedding of Internet nodes based on their pairwise distances: what contributes to the estimation errors? Can such errors be reduced by increasing the dimensionality of embedding? More fundamentally, how do we quantify the suitability of Euclidean embedding? We believe that such a systematic understanding is crucial for charting the future research directions in developing more accurate, efficient and scalable network distance estimation techniques. Our paper is a first attempt in reaching such an understanding, and proposes a simple new hybrid model that combines global Euclidean embedding with local non-Euclidean error adjustment for more accurate and scalable network distance estimation.

The contributions of our paper are summarized as follows. First, by applying the classical scaling and matrix perturbation theory, we establish the (sum of the) magnitude of negative eigenvalues of an appropriately transformed, squared distance matrix as a measure of suitability of Euclidean embedding. In particular, existence of negative eigenvalues with large 
magnitude indicates that the set of nodes cannot be embedded well in a Euclidean space with small absolute errors.

Second, using data from real Internet measurement, we show that the distance matrix of Internet nodes indeed contains negative eigenvalues of large magnitude. Furthermore, we establish a connection between the degree of triangle inequality violations (TIVs) in the Internet distances to the magnitude of negative eigenvalues, and demonstrate that the inaccuracy of Euclidean embedding is caused by a large degree of TIVs in the network distances, which leads to negative eigenvalues of large magnitude. We also show that TIVs cause the embedding schemes to be sub-optimal in that the sum of estimation errors from a host are either positive or negative (far from 0), which means that the estimations are biased.

Third, we show that a majority of TIVs occur due to the nodes that are close-by. By clustering nodes based on their distances, we find that while the distances between the nodes in the different clusters (the inter-cluster node distances) can be fairly well-approximated by the Euclidean distance function, the intra-cluster node distances are significantly more nonEuclidean, as manifested by a much higher degree of TIVs and the existence of negative eigenvalues with considerably larger magnitude. Based on these results, we conclude that estimating network distances using coordinates of hosts embedded in a global Euclidean space is rather inadequate for close-by nodes.

As the last (but not the least) contribution of our paper, we develop a new hybrid model for embedding the network nodes: in addition to a low dimensional Euclidean embedding (which provides a good approximation to the inter-cluster node distances), we introduce a locally determined (non-metric) adjustment term to account for the non-Euclidean effect within the clusters. The proposed hybrid model is mathematically proved to always reduce the estimation errors in terms of stress (a standard metric for fitness of embedding). In addition, this model can be used in conjunction with any Euclidean embedding scheme.

The remainder of the paper is organized as follows. In Section II, we provide a mathematical formulation for embedding nodes in a Euclidean space based on their distances, and apply the classical scaling and matrix perturbation theories to establish the magnitude of negative eigenvalues as a measure for suitability of Euclidean embedding. In Section III, we analyze the suitability of Euclidean embedding of network distances and investigate the relationship between triangle inequality violations and the accuracy. Section IV shows the accuracy of various Euclidean embedding schemes over various real measurement data sets. We show the clustering effects on the accuracy in section $\mathrm{V}$. We describe the new hybrid model for the network distance mapping in Section VI and conclude the paper in Section VII.

\section{EuClideAn Embedding AND Classical ScAling}

In this section we present a general formulation of the problem of embedding a set of points (nodes) into a $r$-dimensional Euclidean space given the pairwise distance between any two nodes. In particular, using results from classical scaling and matrix perturbation theories we establish the (sum of the) magnitude of negative values of an appropriately transformed, squared distance matrix of the nodes as a measure for the suitability of Euclidean embedding.

\section{A. Classical Scaling}

Given only the $n \times n$, symmetric distance matrix $D=\left[d_{i j}\right]$ of a set of $n$ points from some arbitrary space, where $d_{i j}$ is the distance ${ }^{1}$ between two points $\mathbf{x}_{i}$ and $\mathbf{x}_{j}, 1 \leq i, j \leq n$, we are interested in the following problem: can we embed the $n$ points $\left\{\mathbf{x}_{1}, \mathbf{x}_{2}, \ldots, \mathbf{x}_{n}\right\}$ in an $r$-dimensional space for some integer $r \geq 1$ with reasonably good accuracy? To address this question, we need to first determine what is the appropriate dimension $r$ to be used for embedding; given $r$ thus determined, we then need to map each point $\mathbf{x}_{\mathbf{i}}$ into a point $\tilde{\mathbf{x}}_{i}=\left(\tilde{x}_{i 1}, \ldots, \tilde{x}_{i r}\right)$ in the $r$-dimensional Euclidean space to minimize the overall error of embedding with respect to certain criterion of accuracy.

Before we address this problem, we first ask a more basic question: Suppose that the $n$ points are actually from an $r$ dimensional Euclidean space, given only their distance matrix $D=\left[d_{i j}\right]$, is it possible to find out the original dimension $r$ and recover their original coordinates in the $r$-dimensional space? Fortunately, this question is already answered by the theory of classical scaling [10]. Let $D^{(2)}=\left[d_{i j}^{2}\right]$ be the matrix of squared distances of the points. Define $B_{D}:=-\frac{1}{2} J D^{(2)} J$, where $J=I-n^{-1} \mathbf{1 1 ^ { T }}, I$ is the unit matrix and $\mathbf{1}$ is a $n$ dimensional column vector whose entries are all $1 . J$ is called a centering matrix, as multiplying $J$ to a matrix produces a matrix that has 0 mean columns and rows. Hence $B_{D}$ is a doubly-centered version of $D^{(2)}$. A result from the classical scaling theory gives us the following theorem.

Theorem 1: If a set of $n$ points $\left\{\mathbf{x}_{1}, \mathbf{x}_{2}, \ldots, \mathbf{x}_{\mathbf{n}}\right\}$ are from an $r$-dimensional Euclidean space. Then $B_{D}$ is semi-definite with exactly $r$ positive eigenvalues (and all other eigenvalues are zero). Furthermore, let the eigen decomposition of $B_{D}$ is given by $B_{D}=Q \Lambda Q^{T}=Q \Lambda^{1 / 2}\left(Q \Lambda^{1 / 2}\right)^{T}$, where $\Lambda=\left[\lambda_{i}\right]$ is a diagonal matrix whose diagonal consists of the eigenvalues of $B_{D}$ in decreasing order. Denote the diagonal matrix of the first $r$ positive eigenvalues by $\Lambda_{+}$, and $Q_{+}$the first $r$ columns of $Q$. Then the coordinates of the $n$ points are given by the $n \times r$ coordinate matrix $Y=Q_{+} \Lambda_{+}^{1 / 2}$. In particular, $Y$ is a translation and rotation of the original coordinate matrix $X$ of the $n$ points.

Hence the above theorem shows that if $n$ points are from a Euclidean space, then we can determine precisely the original dimension and recover their coordinates (up to a translation and rotation). The contrapositive of the above theorem states that if $B_{D}$ is not semi-definite, i.e., it has negative eigenvalues, then the $n$ points are not originally from an Euclidean space. A natural question then arises: does the negative eigenvalues of $B_{D}$ tell us how well a set of $n$ points can be embedded in a Euclidean space? In other words, can they provide an appropriate measure for suitability of Euclidean embedding? We formalize this question as follows. Suppose the $n$ points are

\footnotetext{
${ }^{1}$ We assume that the distance function $d(\cdot, \cdot)$ satisfy $d(x, x)=0$ and $d(x, y)=d(y, x)$ (symmetry), but may violate the triangle inequality $d(x, z) \leq d(x, y)+d(y, z)$; hence $d$ may not be metric.
} 


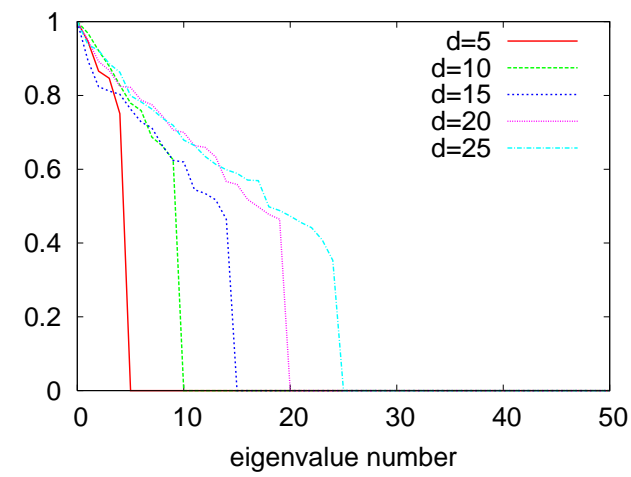

(a) Random points from Euclidean Space

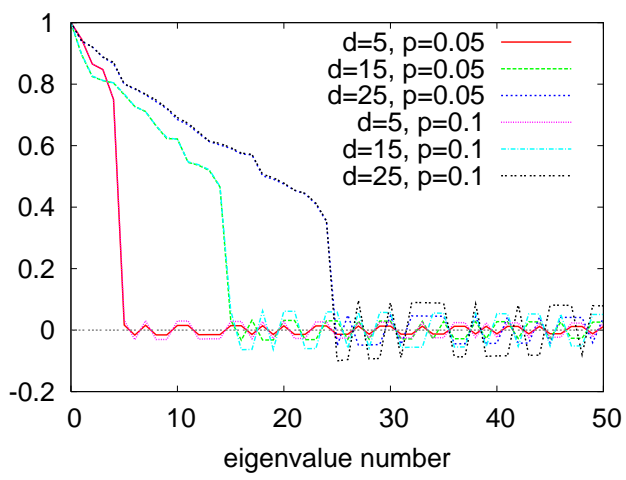

(b) Random points with noise

Fig. 1. Scree plots of the eigenvalues on data sets. Random points are generated in $d$-dimensional Euclidean space. The noise is computed as $d n o i s e_{x y}=$ $d_{x y}+d_{x y} \times f$, where $f$, the noise factor, is uniformly randomly selected from a range of $[0, p) . p=0.05$ and $p=0.1$ are used.

from an $r$-dimensional Euclidean space, but the actual distance $\tilde{d}_{i j}$ between two points $\mathbf{x}_{\mathbf{i}}$ and $\mathbf{x}_{\mathbf{j}}$ is "distorted" slightly from their Euclidean distance $d_{i j}$, e.g., due to measurement errors. Hence, intuitively if the total error is small, we should be able to embed the $n$ points into an $r$-dimensional Euclidean space with small errors. Using the matrix perturbation theory, in the following we show that in such a case the (doubly centered) squared distance matrix must have small negative eigenvalues.

Formally, we assume that $\tilde{d}_{i j}^{2}=d_{i j}^{2}+e_{i j}$, where $\left|e_{i j}\right| \leq$ $\epsilon / n$ for some $\epsilon>0$. Hence $\tilde{D}^{2}:=\left[\tilde{d}_{i j}^{2}\right]=D^{(2)}+E$, where $E:=\left[e_{i j}\right]$. A frequently used matrix norm is the Frobenius norm, $\|E\|_{F}:=\sqrt{\sum_{i} \sum_{j}\left|e_{i j}\right|^{2}} \leq \epsilon$. Then $B_{\tilde{D}}:=$ $-\frac{1}{2} J \tilde{D}^{(2)} J=B_{D}+B_{E}$, where $B_{E}:=-\frac{1}{2} J E J$. It can be shown that $\left\|B_{E}\right\|_{F} \leq \epsilon$. For $i=1,2, \ldots, n$, let $\tilde{\lambda}_{i}$ and $\lambda_{i}$ be the $i$ th eigenvalue of $B_{\tilde{D}}$ and $B_{D}$ respectively, where $\tilde{\lambda}_{1} \geq \cdots \geq \tilde{\lambda}_{n}$ and $\lambda_{1} \geq \cdots \geq \lambda_{n}$. Then the WiedlandtHoffman Theorem [11] states that $\sum_{i=1}^{n}\left(\tilde{\lambda}_{i}-\lambda_{i}\right)^{2} \leq\left\|B_{E}\right\|_{F}^{2}$. Since $\lambda_{i} \geq 0$, we have

$$
\sum_{\left\{i: \tilde{\lambda}_{i}<0\right\}}\left|\tilde{\lambda}_{i}\right|^{2} \leq \sum_{\left\{i: \tilde{\lambda}_{i}<0\right\}}\left(-\tilde{\lambda}_{i}+\lambda_{i}\right)^{2} \leq \sum_{i=1}^{n}\left(\tilde{\lambda}_{i}-\lambda_{i}\right)^{2} \leq\left\|B_{E}\right\|_{F}^{2} \leq \epsilon^{2} .
$$

Hence the sum of the squared absolute values of the negative eigenvalues is bounded by the squared Frobenius norm of the (doubly-centered) error matrix $\left\|B_{E}\right\|_{F}^{2}$, which is the sum of the (doubly-centered) squared errors. In particular, the absolute value of any negative eigenvalue $\left|\tilde{\lambda}_{i}\right|$ is bounded by $\left\|B_{E}\right\|_{F}$. Hence if the total error (as reflected by $\left\|B_{E}\right\|_{F}^{2}$ ) is small and bounded by $\epsilon$, then the negative eigenvalues of $\tilde{B}_{D}$ are also small and their magnitude is bounded by $\epsilon$. Hence the magnitude of negative eigenvalues (and their sum) provides a measure of the suitability of Euclidean embedding: if a set of $n$ points can be well-approximated by a Euclidean space with an appropriate dimension, then their associated doubly-centered squared distance matrix only has negative eigenvalues of small magnitude, if any. On the other hand, the contrapositive of the above proposition leads to the following observation:

Theorem 1: If the doubly-centered squared distance matrix of a set of $n$ points has negative eigenvalues of large magnitude, then the set of $n$ points cannot be embedded into a Euclidean space with a small total error (as measured by
$\left.\left\|B_{E}\right\|_{F}\right)$. In other words, they are less amenable to Euclidean embedding.

In this derivation, we use total error, $\epsilon$. However, the total error can be from only a few distance estimations so that eigenvalue analysis can wrongfully conclude that the Euclidean embedding is not good for this distance matrix. Actually, the meaning of good fitting depends on the objectives of the embedding. Typical objective functions usually try to minimize the total sum of squared absolute errors or relative errors. In such case, even if only a few distances happen to have really high error terms, the errors are distributed to a large number of points because these objective functions tend to prefer many small errors rather than a few large errors. As a consequence, when the total error is high (regardless of whether it is from a few sources or many sources), the embedding is difficult to find the original positions of the points in the Euclidean space. So the eigenvalue analysis is useful to measure the suitability of the Euclidean embedding computed by the embedding schemes of which objective functions are to minimize the total (sum of squared) error.

\section{B. Illustration}

We now generate some synthetic data to demonstrate how classical scaling can precisely determine the original dimensionality of data points that are from a Euclidean space. First, we generate 360 random points in a unit hyper cube with different dimensions and compute the corresponding distance matrix for each data set. Fig. 1(a) shows the scree plot of the eigenvalues obtained using classical scaling. The eigenvalues are normalized by the largest value (This will be the same for the rest of the paper). We see from Fig. 1(a) that the eigenvalues vanish right after the dimensionality of the underlying Euclidean space where the data points are from, providing an unambiguous cut-off to uncover the original dimensionality. We now illustrate what happens when distances among data points are not precisely Euclidean (e.g., due to measurement errors). We add noise to the synthetically generated Euclidean data sets as follows: the noise component in the data is $d \times(1+f)$, where $d$ is the original Euclidean distance and $f$ is a randomly selected number from $(-p, p)$. We use $p=0.05$ and $p=0.1$ for the illustration below. We 


\begin{tabular}{|r|r|r|}
\hline Data Set & Nodes & Date \\
\hline King462 ([12]) & 462 & $8 / 9 / 2004$ \\
\hline King2305 ([13]) & 2305 & 2004 \\
\hline PlanetLab ([14]) & 148 & $9 / 30 / 2005$ \\
\hline Ng02 ([15]) & 19 & May 2001 \\
\hline
\end{tabular}

TABLE I

THE DATA SETS USED IN OUR STUDY. THE NUMBER OF NODES IS CHOSEN TO MAKE THE MATRIX COMPLETE AND SQUARE.

observe in Fig. 1(b) that the first $r$ eigenvalues are positive, and are nearly the same as in the case without noise, where $r$ represents the actual dimension of the data set. Beyond these eigenvalues, we observe only small negative eigenvalues. As the noise increases, the magnitudes of negative eigenvalues increase slightly. It is clear that as the data set deviates from Euclidean more, the magnitudes of the negative eigenvalues become larger.

\section{Suitability of EuclideAn Embedding}

To understand the suitability of Euclidean embedding of network distances, in this section we perform eigenvalue analysis of the distance matrices and investigate how the triangle inequality violations (TIVs) affect the accuracy of the embedding, and thus the suitability of Euclidean embedding for a wide range of data sets.

To be specific, we apply eigenvalue analysis to show that the (doubly-centered, squared) distance matrices of the data sets contain negative eigenvalues of relatively large magnitude. We then attribute existence of the negative eigenvalues of relative large magnitude to the large amount of triangle inequality violations existing in the data sets by showing: i) embedding a subset of nodes without triangle inequality violations in a Euclidean space produces higher accuracy, and the associated distance matrix also contains only negative eigenvalues of much smaller magnitude; and ii) by increasing the degree of TIVs in a subset of nodes of the same size, the performance of Euclidean embedding degrades and the magnitude of the negative eigenvalues also increases.

We use four different data sets, which we refer to as King462, King2305, and PlanetLab, and Ng02, as listed in Table I. The King462 data set is derived from the data set used by Dabek et al. [12] after removing the partial measurements to derive a $462 \times 462$ complete and square distance matrix among 462 hosts from the original 2000 DNS server measurements. Using the same refinement over the data set used in [13], we derive the King2305 data set, which is a $2305 \times 2305$ complete and square distance matrix. PlanetLab is derived from the distances measured among the Planetlab nodes on Sep 30th 2005 [14]. We chose the minimum of the 96 measurement (one measurement per 15 minutes) data points for each measurement between node pairs. After removing the hosts that have missing distance information, we obtain a $148 \times 148$ distance matrix among 148 nodes. The $\mathrm{NgO2}$ data set is obtained from [15] that contains a $19 \times 19$ distance matrix. Even though the number of hosts is small in this data set, we have chosen this data set in order to compare with the results in other papers.

\begin{tabular}{|c|r|r|r|r|}
\hline Data Set & Ng02 & King2305 & King462 & Planetlab \\
\hline fraction & 0.116 & 0.233 & 0.118 & 0.131 \\
\hline \multicolumn{5}{c}{ TABLE II } \\
THE FRACTION OF TIVS OVER ALL TRIPLES OF NODES
\end{tabular}

\section{A. Eigenvalue Analysis}

First, we perform eigenvalue analysis of the doublycentered, squared distance matrix $B_{D}=-J D^{(2)} J$. Fig. 2 shows the scree plot of the resulting eigenvalues, normalized by the eigenvalue of the largest magnitude $\left|\lambda_{1}\right|$, in decreasing order in the magnitude of the eigenvalues. We see that each of the data sets has one or more negative eigenvalues of relatively large magnitude that are at least about $20 \%$ (up to $100 \%$ ) of $\left|\lambda_{1}\right|$, and the negative eigenvalue of largest magnitude is among the second and fourth largest in terms of magnitude). This suggests that the network distances are somewhat less suitable for Euclidean embedding. Hence it is expected that embedding the nodes in a Euclidean space would produce considerable amount of errors.

\section{B. TIV Analysis}

Motivated by earlier studies (e.g., [7]), which show that there is a significant amount of TIVs in the Internet distance measurement, and attribute such TIVs to Internet routing policies $^{2}$, here, we investigate how the amount of TIVs in the data sets affect the suitability and accuracy of Euclidean embedding of network distances. In particular, we establish a strong correlation between the amount of TIVs and the magnitude of negative eigenvalues of the associated distance matrix. First we analyze the amount of TIVs in the four data sets. For each data set, we take a triple of nodes and check whether they violate triangle inequality. We then compute the fraction of such TIVs over all possible triples. Table II shows the results for the four data sets. We see that the fraction of TIVs in the King2305 data set is about 0.23 , while for the other three data sets, it is around 0.12 . Hence the triangle inequality violations are fairly prevalent in the data sets.

To investigate how the amount of TIVs affect the suitability and accuracy of Euclidean embedding - in particular, its impact on the magnitude of negative eigenvalues, we start with a subset of nodes without any triangle inequality violation (we refer to such a subset of nodes as a $T I V$-free set). Ideally we would like this subset to be as large as possible, namely, obtain the maximal TIV-free (sub)set. Unfortunately, finding the maximal TIV-free subset is NP-hard, as is stated in the following theorem (the proof of which is delegated to the appendix).

Theorem 2: Finding the maximal TIV-free set problem is NP-complete.

Hence we have to resort heuristics to find a large TIV-free set. Here we describe three heuristic algorithms. The basic algorithm (referred to as Algo 0 ) is to randomly choose $k$ nodes from a given set of $n$ nodes and check whether any

\footnotetext{
${ }^{2}$ In particular, [7] shows that the Hot Potato Routing policy and the interplay between inter-domain and intro-domain routing can cause TIVs. It also shows that private peering between small ASes is another source of TIVs.
} 


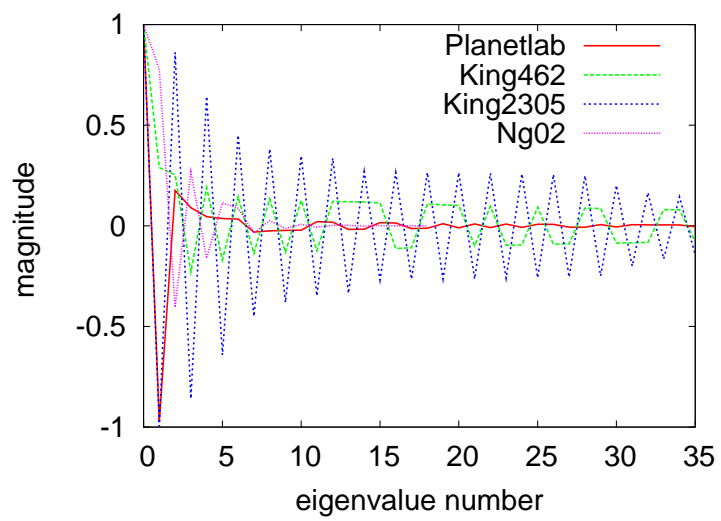

Fig. 2. The eigenvalue scree plot of network distance matrices.

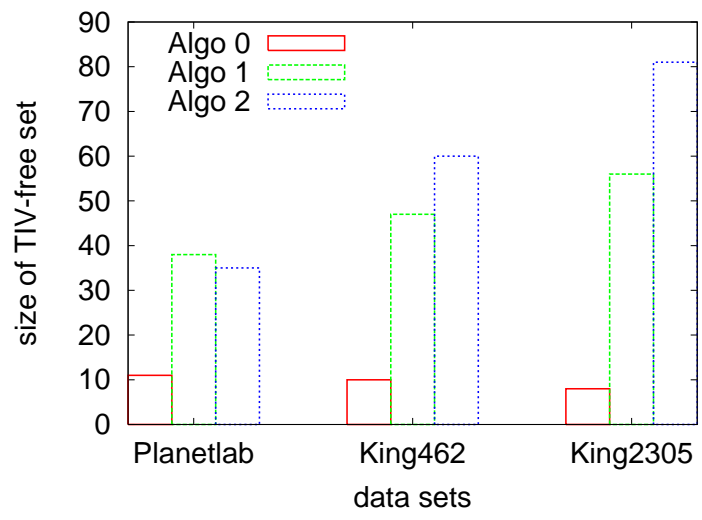

Fig. 3. Performance of the 3 heuristic algorithms.

three nodes of these randomly selected $k$ nodes violates the triangle inequality. If the triangle inequality is violated, the process is repeated again by randomly selecting another set of $k$ nodes. If we find a TIV-free set of size $k$, we increase $k$ by one and try again to attempt to find a larger set. Otherwise the algorithm terminates after a pre-specified number of failed tries, and returns the TIV-free set of size $k-1$.

The second heuristic algorithm (Algo 1) is as follows. We start with a TIV-free set (initialized with two randomly selected nodes). From the remaining node set $C$ (initially with $n-2$ nodes), we then randomly pick a new node and check to see whether it violates the triangle inequality with any two nodes in the existing TIV-free set. If yes, this node is removed from the remaining node set $C$. Otherwise it is added to the TIV-free set (and removed from the remaining node set). The process is repeated until the remaining node set becomes empty.

The third heuristic algorithm (Algo 2) is slightly more sophisticated, and works in a similar fashion as Algo 1, except that we do not choose nodes randomly for consideration. We start with an initial TIV-free set $A$ of two nodes, where the two nodes are chosen such that the pair of nodes has the least number of TIVs with nodes in the remaining node set $C$. Given this pair of nodes, we remove all nodes in the remaining node set $C$ that violate the triangle inequality with this pair of nodes. For each node $c$ in $C$, we compute the number of nodes in $C$ that violates triangle inequality with $c$ and any two nodes in $A$. We pick the node $c$ that has the smallest such number, add it to $A$ and remove it from $C$. We then purge all the nodes in $C$ that violate the triangle inequality with $c$ and any two nodes in $A$. We repeat the above process until $C$ becomes empty.

For the data sets Planetlab, King462 and King2305 (the $\mathrm{NgO2}$ data set is not used since it is too small), the size of largest TIV-free sets found using the three heuristic algorithms is shown in Fig. 3. For each data set, Algo 0 only finds a TIVfree set of about 10 nodes. Algo 2 finds the largest TIV-free sets for the King462 and King 2305 data sets, while Algo 1 finds the largest TIV-free set for the Planetlab data set. For the following analysis, we use the largest TIV-free set found for each data set. Fig. 4(a) shows the scree plot of the eigenvalues for the associated (doubly-centered, squared) distance matrix of the TIV-free node sets. We see that they all have only a small number of negative eigenvalues and the magnitude of all the negative eigenvalues is also fairly small. Comparing with Fig. 2, either the number or the magnitude of negative eigenvalues is significantly reduced.

The embedding accuracy of the TIV-free data sets is shown in Fig. 4(b). The relative errors, which are defined precisely in Section IV-A, are relatively small. For example, for the Planetlab data set, in almost $98 \%$ of the cases, the relative errors are less than 0.2. We see that the Euclidean embedding of the TIV-free sets has a fairly good overall accuracy. However, Fig. 4(b) still shows non negligible errors for the TIVfree data sets. Since multidimensional scaling methods such as GNP can actually embed Euclidean data set without any error, this means that the errors of the TIV-free data set embedded by GNP are truly from the non-Euclidean characteristics of Internet routing. Actually, it is well known that the nonEuclidean metric space such as the shortest path routing is hard to embed into a low-dimensional Euclidean space without distortions or errors ([16]).

\section{Correlation between Negative Eigenvalues and Amount of TIVs}

Next, we show how the amount of TIVs in a data set contributes to the magnitude of negative eigenvalues, thereby the suitability and accuracy of Euclidean embedding. We use the King2305 data set as an example. The largest TIV-free set we found has 81 nodes. We fix the size of the node sets, and randomly select six other node sets with exactly 81 nodes, but with varying amount of TIVs. The scree plots of the eigenvalues for the six node sets are shown in Fig. 5(a), and the cumulative relative error distributions of the corresponding Euclidean embedding are shown in Fig. 5(b). We see that with the increasing amount of TIVs, both the magnitude and number of negative eigenvalues increase. Not surprisingly, the overall accuracy of the Euclidean embedding degrades. In fact, we can mathematically establish a relation between the amount of TIVs and the sum of squared estimation errors as follows.

Lemma 1: If the distances $t_{a}, t_{b}, t_{c}$ among 3 nodes violate the triangle inequality, i.e., $t_{c}>t_{a}+t_{b}$, the minimum squared estimation error of any metric (thus Euclidean) embedding of the 3 nodes is $\frac{\left(t_{c}-t_{a}-t_{b}\right)^{2}}{3}$. 


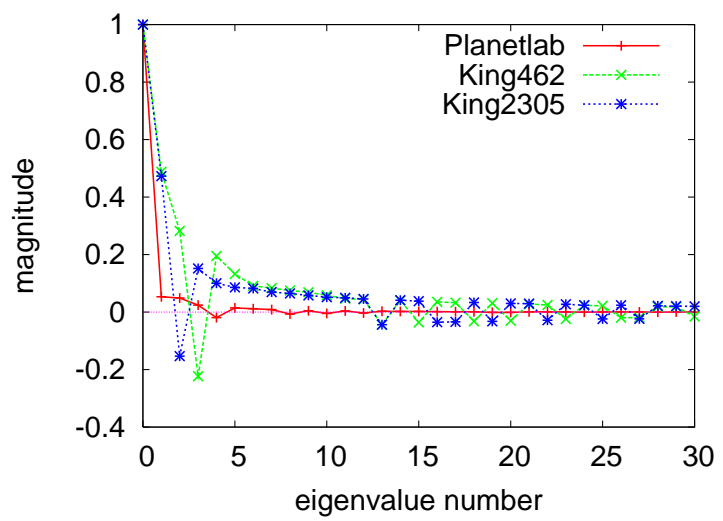

(a) Eigenvalues

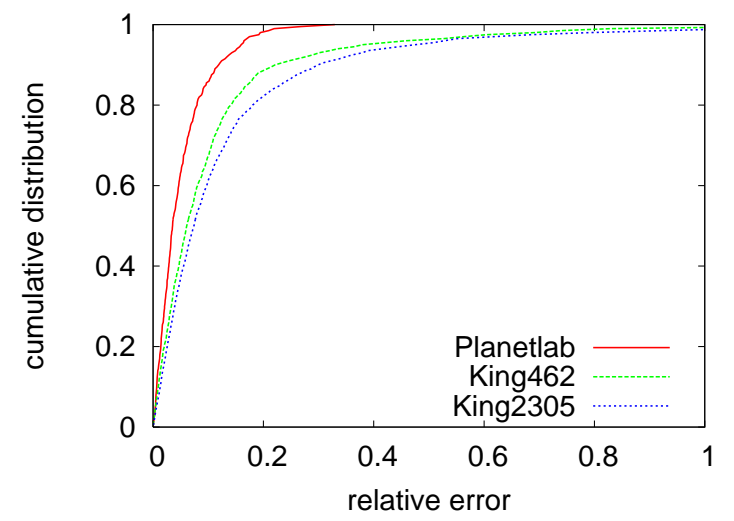

(b) Relative Errors

Fig. 4. Performance of embedding the TIV-free node sets using GNP.

Theorem 3: The sum of squared estimation errors of any Euclidean embedding of $n$ nodes is at least $\frac{1}{3(n-2)} \sum_{t \in V}\left(t_{c}-t_{a}-t_{b}\right)^{2}$, where $V$ is the set of triples that violate triangle inequality, $t_{a}, t_{b}$, and $t_{c}$ are the 3 distances of a triple $t \in V$, and $t_{c}>t_{a}+t_{b}$.

The proofs are delegated to the appendix. Theorem 3 shows that as the amount of TIVs increases, the sum of the squared estimation errors also increases. A similar result can also be established for the sum of squared relative errors, the details of which are omitted here. As an aside, we note that this theorem holds not only for Euclidean distance function, but also for any metric distance function where the triangle inequality property is required. However, it should be noted that the lower bound computed in Theorem 3 is loose in some cases. For example, the lower bound for the TIV-free data set is 0 , but the embedding has non-negligible errors. Nonetheless, Theorem 3 sheds new lights on the relationship between the accuracy and the amount of TIVs.

\section{Euclidean Embedding of Network Distances}

In this section, we examine the accuracy of Euclidean embedding of network distances for a wide range of data sets. We consider five different metrics that we believe are useful for a variety of delay sensitive applications.

\section{A. Metrics for Goodness-of-Embedding}

We consider four performance metrics, namely, stress, (cumulative) relative errors, relative rank loss (RRL), and closest neighbor loss $(C N L)$ that have been introduced across various studies in the literature (e.g., [2], [3], [4], [8]), as well as a new fifth metric skewness we introduce in this paper to gauge whether an embedding is more likely to over- or underestimate the distances between the nodes. These five metrics are formally defined as follows:

- Stress: This is a standard metric to measure the overall fitness of embedding, originally known as Stress-1 [10]:

$$
\text { Stress-1 }=\sigma_{1}=\sqrt{\frac{\sum_{x, y}\left(d_{x y}-\hat{d}_{x y}\right)^{2}}{\sum_{x, y} d_{x y}^{2}}},
$$

where $d_{x y}$ is the actual distance between $x$ and $y$, and $\hat{d}_{x y}$ is the estimated one.

- Relative error [2]: This metric is introduced in [2] that is defined as follows: for each pair of nodes $x$ and $y$, the relative error in their distance embedding is given by $r e_{x y}:=\frac{\left|d_{x y}-\hat{d}_{x y}\right|}{\min \left(d_{x y}, \hat{d}_{x y}\right)}$. Note that the denominator is the minimum of the actual distance and the estimated one $\mathrm{e}^{3}$. The cumulative distribution of relative errors, $r_{x y}$ 's, provides a measure of the overall fitness of the embedding.

- Relative rank loss (RRL) [8]: RRL denotes the fraction of pair of destinations for which their relative distance ordering, i.e., rank in the embedded space with respect to a source has changed compared to the actual distance measurement. For example, for a given source node, we take a pair of destinations and check which one is closer to the source in the real distances and the estimated distances. If the closest one is different, then the relative rank is defined to be lost. We compute the fraction of such relative rank losses for each source, and plot the cumulative distribution of such rank losses among all sources as a measure of the overall fitness of the embedding.

- Closest neighbor loss (CNL) [8]: For each source, we find the closest node in the original data set and the embedded space If the two nodes are different, the closest neighbor is lost. The $C N L$ metric is then defined as the fraction of sources that have the closest neighbor lost. As an extension to the original CNL metric in [8], we introduce a margin parameter $\delta$ : if the closest neighbor nodes in the original data set and the embedded space are different, but the distance between the two nodes in the embedded space is within $\delta \mathrm{ms}$, we consider it as a nonloss; only if the distance between the two is more that $\delta$ $\mathrm{ms}$, we consider it as a closest neighbor loss. Hence with $\delta=0$, we have the original CNL. We expect that as $\delta$ increases, the CNL metric decreases.

- Skewness: We introduce a new metric skewness to gauge whether an embedding is more likely to over- or under-

\footnotetext{
${ }^{3}$ In some literature, instead of $\min \left(d_{x y}, \hat{d}_{x y}\right), d_{x y}$ is used. This usually produces smaller relative errors.
} 


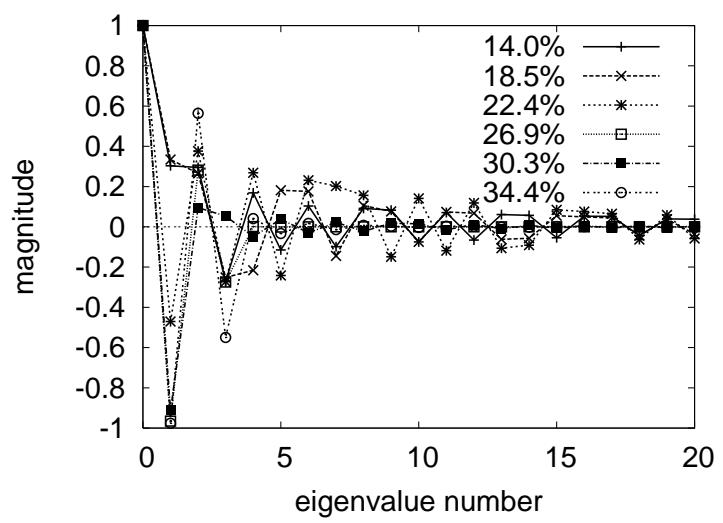

(a) Eigenvalues

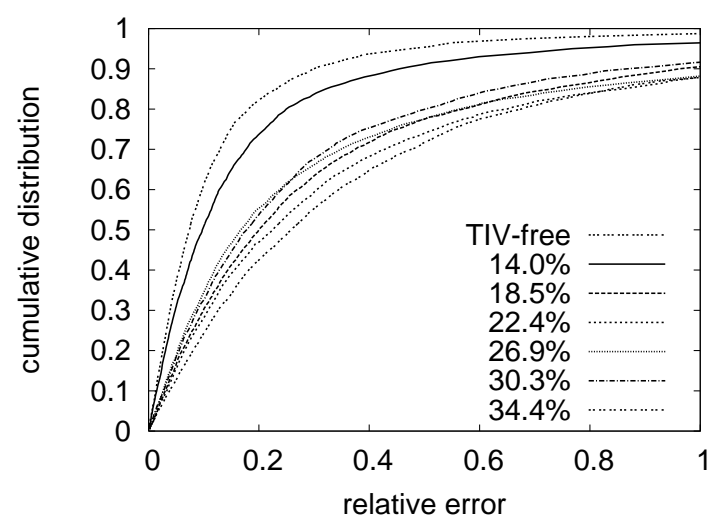

(b) Relative error

Fig. 5. Eigenvalue scree plots and cumulative distributions of relative errors of node sets with increasing fraction of TIVs.

estimate the distances between the nodes. For each node $x$, we define the skewness of embedding with respect to node $x$ as follows: $s_{x}=\sum_{y \neq x}\left(d_{x, y}-\hat{d}_{x, y}\right) /(n-1)$, where $n$ is the total number of nodes. In other words, $s_{x}$ is the average of the embedding errors between the real distances and the estimated distances between node $x$ to all the other nodes. Clearly, when $s_{x}$ is a large positive, the embedding method tends to under-estimate the distances between node $x$ to other nodes, and if it is a large negative, it tends to over-estimate the distances between node $x$ to other nodes. Note that $s_{x}=0$ does not mean that there is no error, but that the under-estimates and over-estimates are "balanced", i.e., canceled out. The distribution of skewnesses of all nodes then provides us with a measure whether a given embedding method tends to under- or over-estimate the real distances among the nodes.

\section{B. Performance of Euclidean Embedding}

We apply three most commonly used embedding methods proposed in the literature - namely, GNP [2], Virtual Landmark [3], [4] and Vivaldi [5] - to the four data sets, and compute their corresponding embedding errors as measured using the aforementioned five metrics. Following the results in [2], [3], [4], we choose 7 as the dimension of Euclidean embedding for the three embedding methods: GNP, Virtual Landmark (VL) and Vivaldi. More specifically, for the GNP and VL embedding methods, we use 20 landmarks randomly selected from the data set for computing the 7-dimensional Euclidean embedding. For the Vivaldi embedding methods, for each node, 20 neighbors are randomly selected and used for computing the 7-dimensional Euclidean embedding plus the height vector ${ }^{4}$. For the purpose of comparison and to eliminate the effect of landmark selection in the Virtual Landmark embedding method, we also use all the nodes as landmarks to compute the 7-dimension Euclidean embedding ${ }^{5}$ - this is referred to as $V L-A L L$ in the figures that follow.

\footnotetext{
${ }^{4}$ We used the Vivaldi implementation from [17]

${ }^{5}$ Using all the nodes as landmarks (or "neighbors" in the case of Vivaldi) is only computationally feasible for the Virtual Landmark embedding method, not for GNP and Vivaldi!
}

Fig. 6(a) shows the resulting overall stress of embedding using the four embedding methods, GNP, VL, VL-ALL and Vivaldi. Except for the King2305 data set, we see that the overall stress ranges from 0.2 to 0.5 , which indicates that on the average the estimations deviate from the original distances from $20 \%$ to $50 \%$. For the King2305 data set, the overall stress is much larger (above 0.9) for all three methods. This is possibly due to the fact that in the King2305 data set, there are quite a few links with more than 90 seconds RTT which may produce many outliers that can significantly affect the overall stress - this is a major shortcoming of the stress metric 1 . Note that as the $\mathrm{NgO2}$ data set has only 19 nodes, the result for VL and Vivaldi are not available since there are fewer nodes than the required number of landmarks and neighbors (20 nodes). Fig. 6(b) and Fig. 6(c) show the cumulative distributions of relative errors using GNP, VL, VL-ALL and Vivaldi for the data sets King462 and PlanetLab, respectively. We see that all the embedding methods produce a relative error less than 0.5 for more than $75 \%$ (up to $90 \%$ in the case of GNP and Vivaldi) of the estimates.

Fig. 7(a) and Fig. 7(b) show the cumulative distributions of relative rank losses using GNP, VL, VL-ALL and Vivaldi for the data sets King462 and Planetlab, respectively. We see that for all the four methods, more than $70 \%$ of the sources have a fraction of relative rank losses (RRL) less than 0.3 - in other words, with respect to these sources, fewer than $30 \%$ of destination pairs have a different rank order in the embedded space from that in the original data set.

In term of the CNL metric, from Fig. 8(a) and Fig. 8(b) we see that as the margin parameter $\delta$ increases, the fraction of closest neighbor losses (CNL) improves for all embedding methods: embedding the Planetlab nodes in a Euclidean space using GNP, $60 \%$ to $70 \%$ of the sources have a different closest neighbor node in the embedded space (i.e., when $\delta=0$ ), but for only about $20 \%$ of the sources, the closest neighbor node in the original data set is more than $15 \mathrm{~ms}$ (i.e., $\delta=15 \mathrm{~ms}$ ) away from the closest neighbor node in the embedded space. The CNLs of VL, VL-ALL, and Vivaldi lie above that of GNP.

Finally, to compare the skewness of the embedding methods, we use the King 462 data set as a representative example. Fig. 9 shows the results for three embedding methods: GNP, VL-ALL 


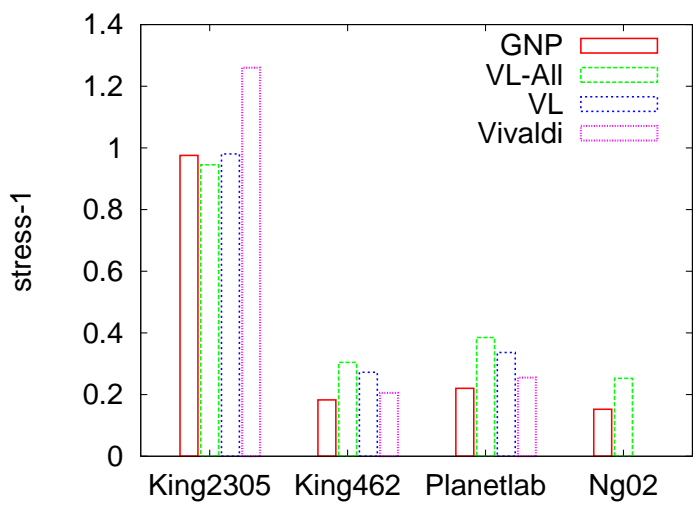

(a) Stress

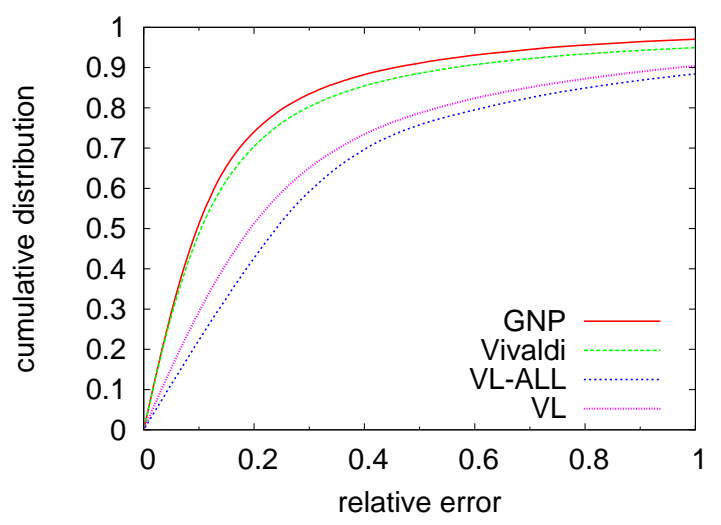

(b) Relative Error with King462

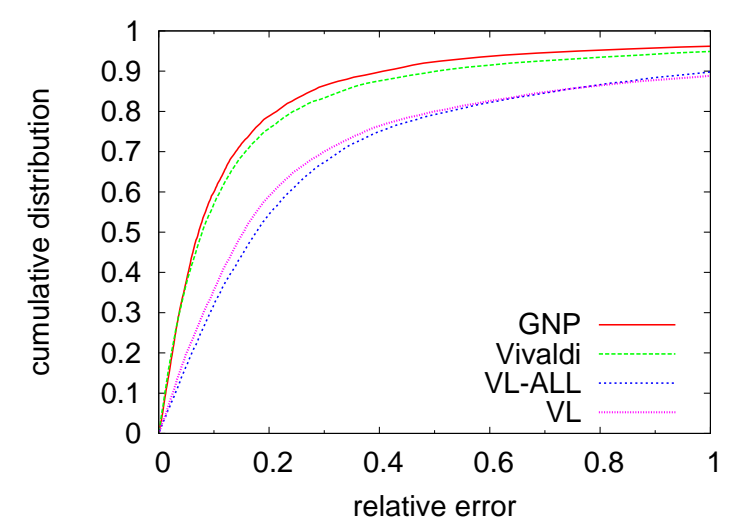

(c) Relative Error with Planetlab

Fig. 6. Performance Embedding. The number of dimensions is 7.

and Vivaldi (to avoid cluttering, we did not include the result for VL in the figure, as it has slightly worse performance than that of VL-ALL). To plot the distribution of the skewness $s_{x}$ 's, we use bins of size of $10 \mathrm{~ms}$ that are centered at multiple of $10 \mathrm{~ms}$, such as $[-15,-5),[-5,5)$, and $[5,15)$. As can be seen in the figure, the GNP and Vivaldi embedding method have the best performance, as the highest fraction of skewness values of the nodes fall within $[-5,5)$, and the majority of the values are within the interval $[-5,15)$. In contrast, however, the skewness values of VL-ALL are more widely spread, indicating that for a fraction of nodes, it tends to either under- or over-estimate their distances to other nodes.

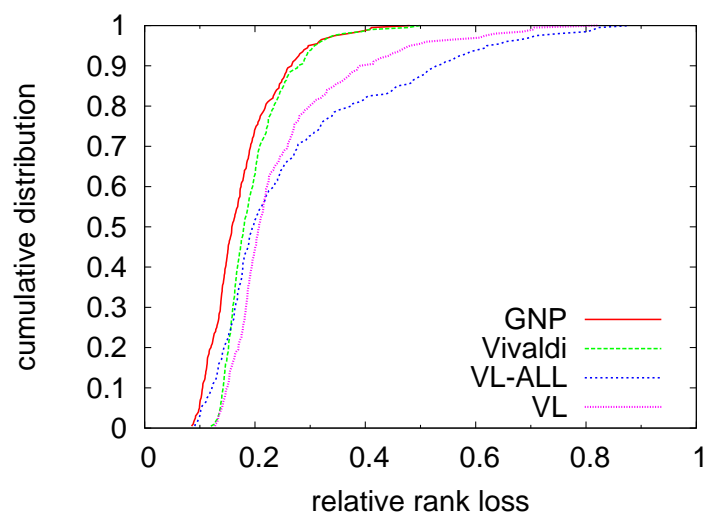

(a) Relative Rank Loss with King462

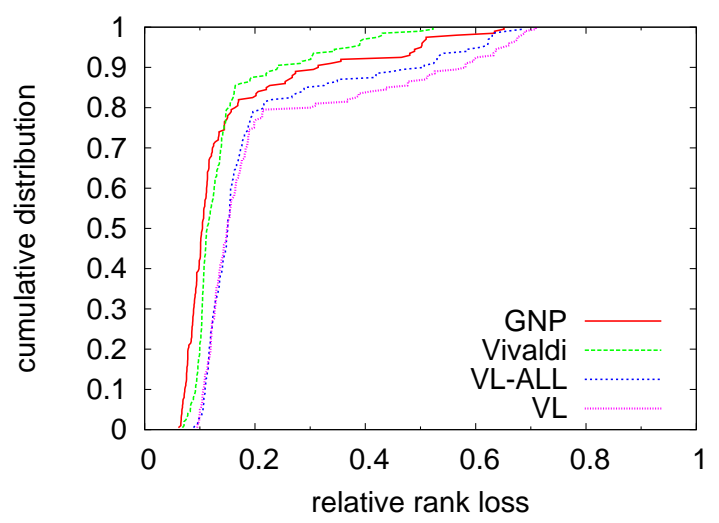

(b) Relative Rank Loss with Planetlab

Fig. 7. The cumulative distribution of Relative Rank Loss.

\section{LOCAL Non-EuCLIDEAN EFFECT}

In this section, we dissect the data set further to find out whether the inherent clustering structure of Internet hosts contribute to the errors in the Euclidean embedding - in particular, what kind of nodes are likely to contribute to the higher degree of TIVs, and whether increasing the dimension of the embedding helps improve the embedding performance.

The hosts in the Internet are clustered due to many factors such as geographical location, network topology and routing policies. This clustering causes many hosts to have short distances among themselves, while far longer distances to other hosts. To investigate the effect of host clustering on embedding accuracy, we first identify clusters within the network distances. For this, we apply the spectral clustering algorithm [18] to the King462 data set with the outliers ${ }^{6}$ removed. In this experiment, 28 nodes out of 462 are removed. The algorithm ${ }^{7}$ obtains four clusters for the King 462 data set. We use a gray scale plot to show the existence of the clusters in the King462 data set with the outliers removed.

\footnotetext{
${ }^{6}$ Outliers are defined as those nodes, the distance of which to the 8th nearest nodes are larger than a threshold. The reason to choose 8 th node is because we want the node to have at least a certain number of neighbors (in this paper, the number is 8) within the threshold.

${ }^{7}$ The algorithm takes as input a parameter $K$, the number of clusters, and produces up to $K$ as a result. We have experimented with $K=3$ to 7 , and the algorithm in general produces 3-4 "relatively big" clusters for the three data sets King462, King2305 and PlanetLab.
} 


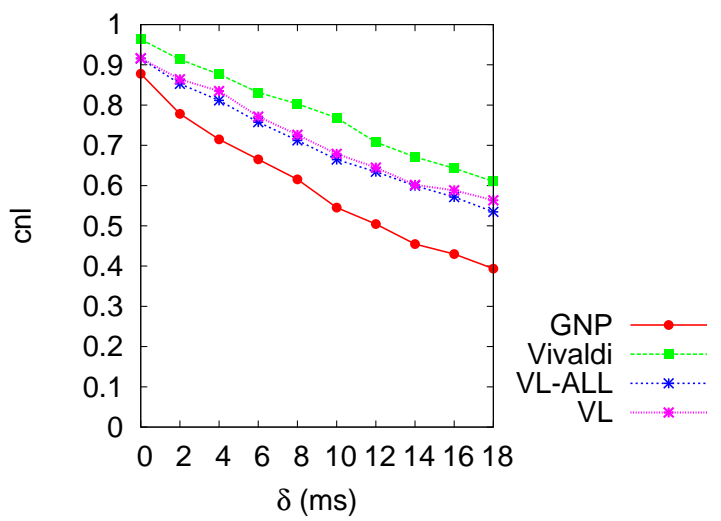

(a) Closest Neighbor Loss with King462

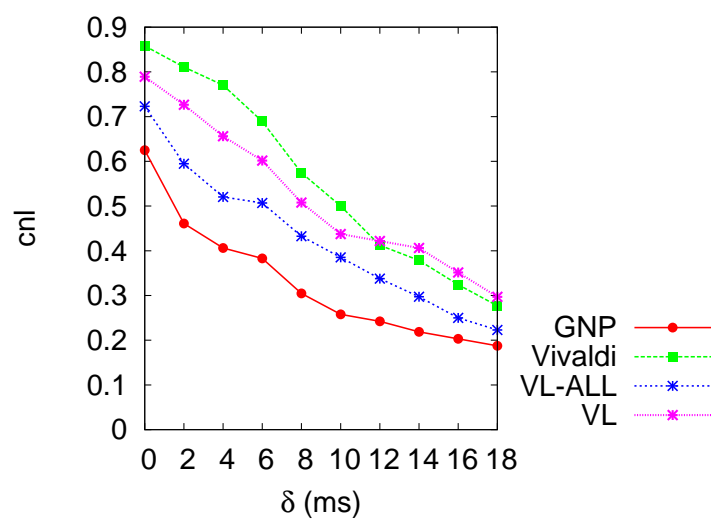

(b) Closest Neighbor Loss with Planetlab

Fig. 8. Closest Neighbor Loss.

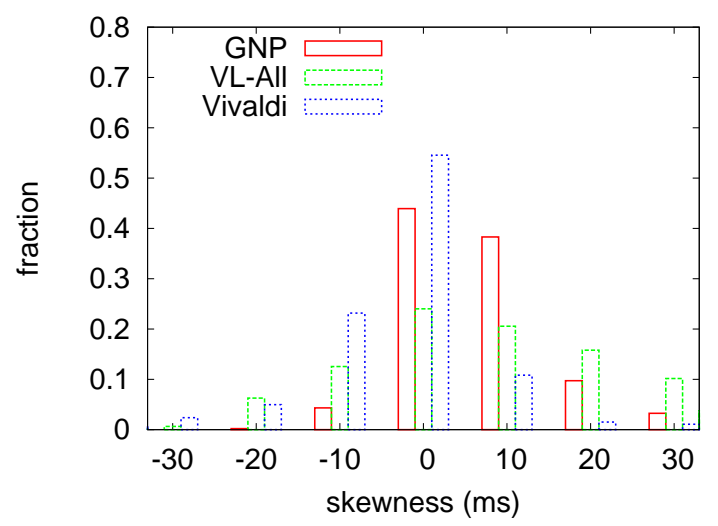

Fig. 9. Distributions of the skewness $s_{x}$ under GNP, VL-ALL and Vivaldi for the King462 data set.

The square image in Fig. 10 is a graphical representation of the King462 distance matrix. In the square image, the vertical axis represents the source nodes and the horizontal axis represents the target nodes. The nodes are sorted by their clusters in such a way that the nodes in cluster 1 appear first, followed by the nodes in cluster 2 , and so on. The nodes in the vertical and horizontal axes are in the same order. Each point, $(x, y)$, represents the distance between the corresponding two nodes, $x$ and $y$.

The distance is represented in a gray scale: white color

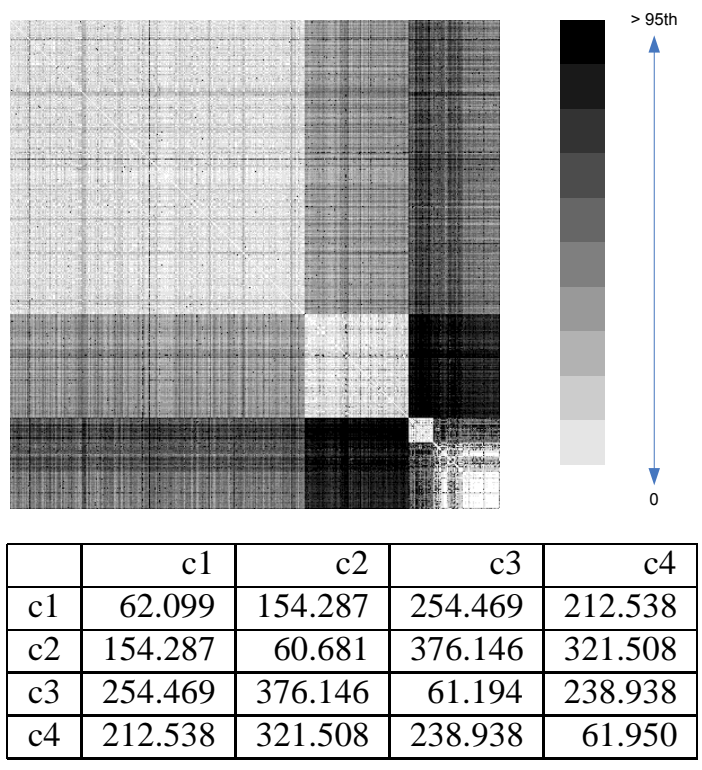

Fig. 10. Distances between each pair of nodes in King462 data set after removing outliers. White represents distance 0 and black represents 95th percentile or higher distances. Median distances (in ms) among the nodes of the intra and inter clusters are shown in the table.

represents distance 0 and black color represents the distance larger than the 95th percentile distance. The interval between 0 and the 95th percentile distance is divided into 10 gray scales (with a total of 11 gray scales), with increasing darkness from white to black (beyond the 95th percentile distance). We can clearly see the four white blocks on the diagonal, each of which represents the distance matrix of each cluster. The table in Fig. 10 shows the median distances between the nodes within and across the four clusters in a numeric form. As is expected, the intra-cluster median distances shown in the diagonal entries of the table are much smaller than the intercluster median distances.

To illustrate the characteristics of the individual clusters, in Fig. 11, we show the eigenvalue scree plots of the distance matrices obtained from the 4 clusters in the King 462 data set. Compared to Fig. 2, we see that the magnitudes of the negative eigenvalues of the clusters are larger than those of the whole data set. The "non-Euclidean-ness" amplifies within each cluster. It suggests that the intra-cluster distances are much harder to embed into the Euclidean space. This can be easily observed by looking at the relative errors of the embedding. Fig. 12 shows the relative errors in a gray scale matrix for the King462 data set, where VL-ALL is used for the embedding. The pure black color represents the relative error of 1.0 or larger, and 10 gray scales are used for relative errors between 0 and 1 . We see that the relative errors of the intra-cluster estimations are larger than those of inter-cluster estimations.

We next examine which nodes are more likely to contribute towards the TIVs. As we shall illustrate next, the high errors in the intra-cluster distance estimation and the large magnitudes of the negative eigenvalues can be explained by the varied number of TIVs over the different distances. Intuitively, a TIV is likely to occur if the distance between two nodes is very 


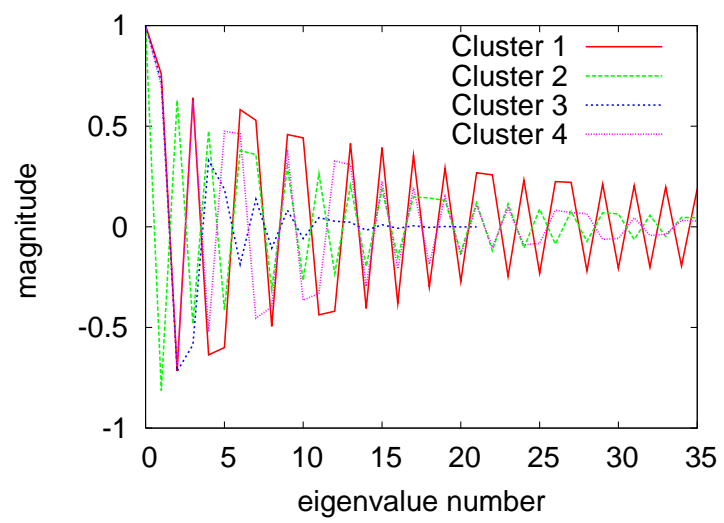

Fig. 11. Scree plot of the eigenvalues of CS on the 4 clusters of the King 462 data set after removing 28 outliers : Cluster 1 (261 nodes), Cluster 2 (92 nodes), Cluster 3 (22 nodes), and Cluster 4 (59 nodes).

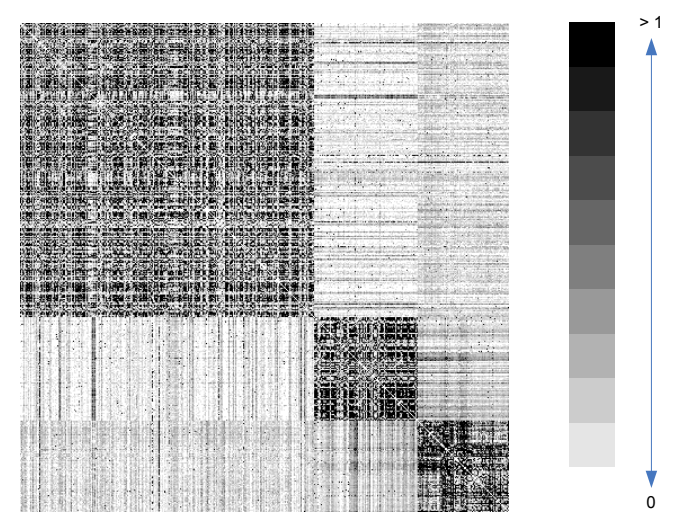

Fig. 12. Relative errors between each pair of nodes in King 462 data set without outliers. White represents relative error 0 and black represents relative error 1 or larger. Virtual Landmark method with 7 dimension is used.

short or very large compared to the other two distances for a given triple of three nodes. Using this intuition we proceed with our data analysis as follows: we divide the distances into 6 intervals: [0ms, 25ms), [25ms, 50ms), [50ms, 100ms), $[100 \mathrm{~ms}, 200 \mathrm{~ms}),[200 \mathrm{~ms}, 400 \mathrm{~ms})$, and $[400 \mathrm{~ms}, \infty)$. We group all the pairs of nodes by their distance intervals. Then, for each pair of nodes, we compute the fraction of TIVs in conjunction with the rest of the nodes, i.e. we count how many nodes violate triangle inequality with the given pair. Finally, we compute the average of the fractions of all the pairs in each interval. Fig. 13 shows the average fraction of TIVs in each distance interval. We observe that higher fractions of TIVs occur in the intervals $[0,25 \mathrm{~ms})$ and $[400, \infty)$ compared to other intervals. Since the fractions of pairs in $[400, \infty)$ are quite small in all the data sets, reducing the errors in short distance estimations is thus much more crucial for the overall performance of embedding.

The above analysis illustrated that the distances among the inter-cluster nodes are more likely to be better approximated by their Euclidean coordinates, whereas Euclidean embedding of nodes within a cluster would likely provide a poor estimate of their distances. This seems to suggest that there is much stronger local "non-Euclidean effect" on the network distances. By local non-Euclidean effect, we mean that the

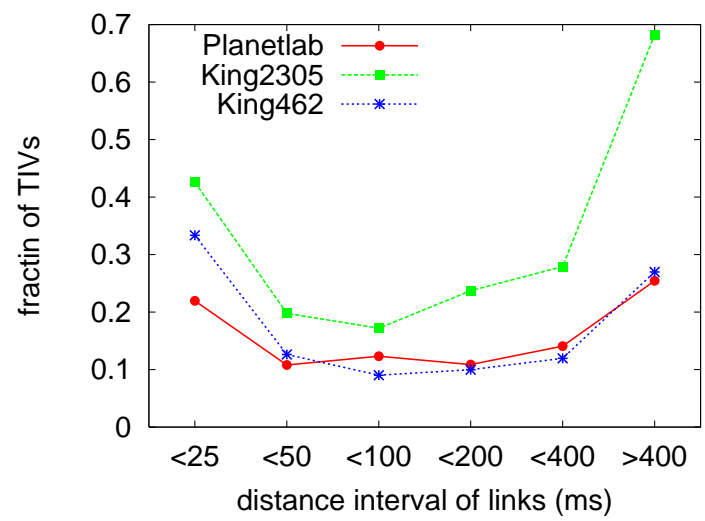

Fig. 13. Average fraction of TIVs at each distance interval.

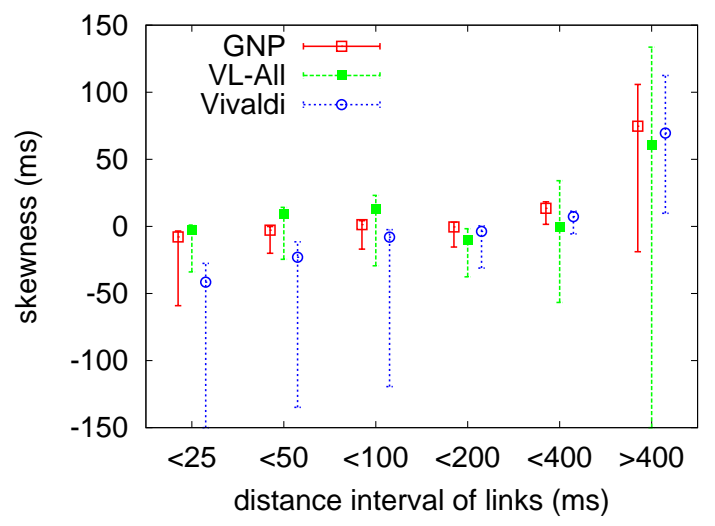

Fig. 14. The 30th, 50th, and 70th percentiles of the skewness measures at each distance interval. The data set is King462.

embedding of local (short) distances into a Euclidean space is much harder than global (long) distances.

The "local non-Euclidean" effect can be also illustrated using the skewness metric. For each node, we compute its skewness to the nodes within the intervals mentioned in the previous sections. Then we plot the 30th percentile, the median, and the 70th percentile of the skewness measures of all nodes using GNP, VL-ALL and Vivaldi. As can be seen in Fig. 14, more skewnesses exist in the intervals $[0,25 \mathrm{~ms})$ and $[400, \infty)$ compared to other intervals. Furthermore, for short distances, the skewness measures are likely to be negative and for large distances, the skewness measures are likely to be positive. Since each of the embedding methods tries to embed the nodes of TIVs in a Euclidean space by minimizing an error function, it is natural to lengthen the short distances and to shorten the long distances. This observation is also evident in the proof of Lemma 1 using the stress error function.

Furthermore, we have examined the impact of increasing dimension and using non-Euclidean distance functions such as the Minkowski $p$-norm on the accuracy of the estimation. Similar to the result in other papers such as [2], increasing dimension does not increase the accuracy and the Minkowski $p$-norm does not help, either. We omit the result due to the page limitation. 


\section{A HYBRID MODEL FOR LOCAL AdJUSTMENT}

The results from previous sections show that the existence of TIVs highly affects the accuracy of the Euclidean embedding (for that matter, any metric embedding). In particular, Euclidean embedding is fairly good at estimating network distances between nodes that are relatively far away (in different clusters), whereas it is rather poor at estimating local network distances (distance between nodes within a cluster). These observations inspire us to develop a hybrid embedding model which incorporates a (non-Euclidean) localized adjustment term (LAT) into the distance estimation. We show that using only a 2-dimensional Euclidean embedding plus the localized adjustment terms, we can obtain better performance than a pure Euclidean embedding with 7 dimensions.

\section{A. The Hybrid Model}

The basic ideas behind our hybrid model are as follows: we first embed the network distances in a Euclidean space of $d$ dimensions, and then for each node we compute an adjustment term to account for the (local) non-Euclidean effect. Hence in our hybrid model, each node $x$ has a $d$-dim Euclidean coordinate, $\left(x_{1}, x_{2}, \ldots, x_{d}\right)$, and a (non-Euclidean) adjustment $e_{x}$ : we use $\left(x_{1}, x_{2}, \ldots, x_{d} ; e_{x}\right)$ to denote the total "coordinate" of node $x$. The distance $d_{x y}$ between two nodes $x$ and $y$ is then estimated by $\hat{d}_{x y}:=d_{x, y}^{E}+e_{x}+e_{y}$, where $d_{x, y}^{E}=\sqrt{\sum_{k=1}^{d}\left(x_{k}-y_{k}\right)^{2}}$ is the Euclidean distance between $x$ and $y$ in the embedded $d$-dim Euclidean space. At the first glance, $e_{x}$ may look similar to the height vector in Vivaldi system [5], but actually it is quite different as will be discussed later in this section. The key question in this model is how to define and determine $e_{x}$ for each node $x$. Ideally, we would like $e_{x}$ to account for the "non-Euclidean" effect on the distance estimation errors to nodes within its own cluster. However, this requires us to know which cluster node $x$ is in as well as the other nodes in its cluster. For simplicity, we derive $e_{x}$ using all nodes as follows. We first compute $\epsilon_{x}$, which minimizes the error function $E(x)=\sum_{y}\left(d_{x y}-\left(d_{x y}^{E}+\epsilon_{x}\right)\right)^{2}$, where $d_{x y}$ is the actual distance between $x$ and $y$. It can be shown that the optimal $\epsilon_{x}$ is given by the average error in estimation:

$$
\epsilon_{x}=\frac{\sum_{y}\left(d_{x y}-d_{x y}^{E}\right)}{n} .
$$

We then set $e_{x}$ to the half of $\epsilon_{x}$, namely, $e_{x}=\epsilon_{x} / 2$. In other words, $\hat{d}_{x y}$ can be re-written as $d_{x, y}^{E}+\frac{\left(\epsilon_{x}+\epsilon_{x}\right)}{2}$. In short, we adjust the Euclidean estimation by the average of the two error terms of $x$ and $y$. We have the following theorem that establishes the advantage of the hybrid model. The proof sketch is provided in the appendix.

Theorem 4: The hybrid model using a $d$-dim Euclidean space and the adjustment term defined above reduces the squared stress of a pure $d$-dim Euclidean embedding by

$$
\frac{4 n \sum_{x} e_{x}^{2}+2 n^{2} \operatorname{Var}\left(e_{x}\right)}{\sum_{x, y} d_{x y}^{2}} \geq 0,
$$

where $\operatorname{Var}\left(e_{x}\right)=\sum_{x} e_{x}^{2} / n-\left(\frac{\sum_{x} e_{x}}{n}\right)^{2}$.
Hence the larger the individual adjustment term $\left|e_{x}\right|$ (thus the average estimation error for each node $x$ using the pure Euclidean embedding), the more performance gain the hybrid model attains. It should be noted that $e_{x}$ can be positive or negative ${ }^{8}$.

In (2), $e_{x}$ is determined by the measurement to all the other nodes in the system. In practice, however, this is not feasible nor scalable. Instead, we compute $\tilde{e}_{x}$ based on sampled measurements to a small number of randomly selected nodes. Let $S$ denote the set of randomly sampled nodes. Then

$$
\tilde{e}_{x}=\frac{\sum_{y \in S}\left(d_{x y}-d_{x y}^{E}\right)}{2|S|} .
$$

Hence in practice the hybrid model works as follows: a) A number of landmarks are pre-selected and perform distance measurements among themselves to obtain a distance matrix. Using either Virtual Landmark or GNP, a $d$-dim Euclidean embedding of the landmarks is obtained and their coordinates are determined. b) Each node $x$ measures their distance to the landmarks and computes its d-dim Euclidean coordinate $\left(x_{1}, x_{2}, \ldots, x_{d}\right)$; it then measures its distance to a small number of randomly selected nodes, and computes $\tilde{e}_{x}$ using eq. (3).

Note that in a sense the adjustment term is similar to the "height vector" introduced in Vivaldi [5]. However, there are several key differences. First of all, the computation of the local adjustment term is very simple, and does not depend on the adjustment term of other nodes. Hence it does not require any iterative process to stabilize the adjustment term. In contrast, in Vivaldi - partly due to its distributed nature a small change in the height vector of a node would affect the height vectors of the other nodes, and requires an iterative process to stabilize the height vectors of all nodes. Second, the local adjustment terms provably improve the performance of network distance embedding, as shown in the above theorem. Another good feature of the local adjustment term is that it can be used with any other schemes, not just the coordinate based schemes. As long as $d_{x y}^{E}$ is the estimated distance based on the original scheme, the adjustment term can be computed as described above. In this sense, LAT is an option that can be used in conjunction with other schemes rather than a totally new scheme. Note that LAT can be used even with Vivaldi.

\section{B. Evaluation}

We evaluate the performance gain obtained by using the localized adjustment term (LAT) option in network distance embedding. For this purpose, we compare the stress of the VL-ALL method without LAT and the VL-ALL method with LAT, where the local adjustment term is computed using all the nodes. We vary the number of dimensions from 2 to 7 . As can be seen in Fig. 15, the use of adjustment term (keys with LAT) reduces the stress significantly compared to the VLAll without LAT. In particular, when the original Euclidean embedding has high stress (large error), the reduction of

\footnotetext{
${ }^{8}$ It is possible that the estimated distance is negative due to negative LAT. In this case, we use the estimation of the Euclidean part as the estimated distance.
} 


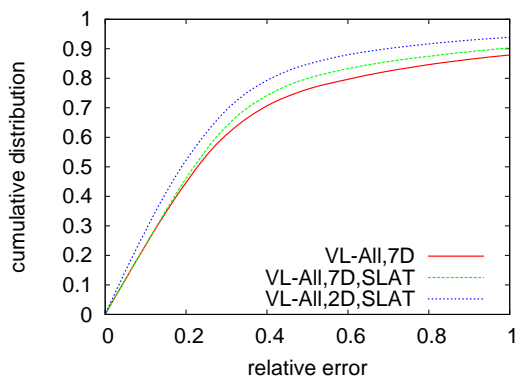

(a) Relative Error

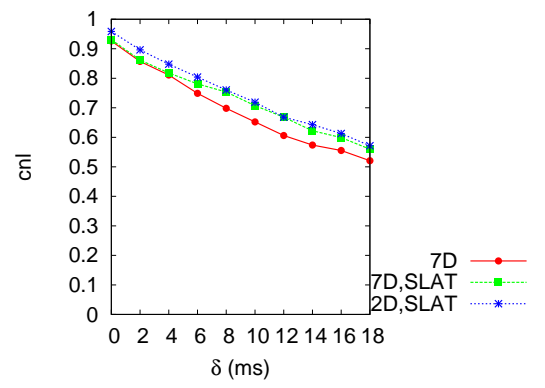

(b) $\mathrm{CNL}$

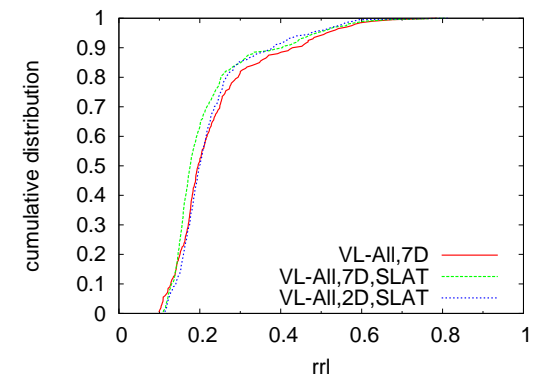

(c) RRL

Fig. 16. The performance of VL-All method with SLAT on King462 data set.

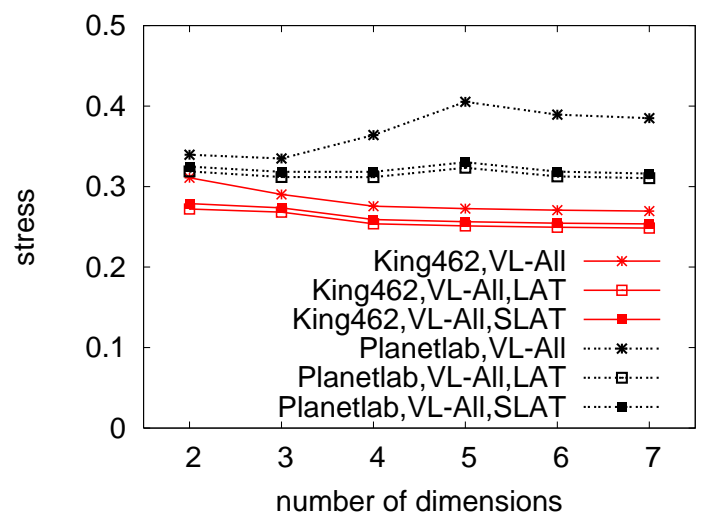

Fig. 15. Stress of Virtual landmarks method over the number of dimensions. Both LAT and SLAT options are shown together.

stress is significant, which is expected from Theorem 4. Next, we evaluate the performance of LAT using only a small number of randomly selected nodes as in eq.(3); we call this option "SLAT (Sampled LAT)". Fig. 15 shows the stress of embedding using SLAT (keys with SLAT) over different number of dimensions, where the adjustment term is computed using the measurement to 10 randomly selected nodes. We see that the performance between LAT and SLAT are very close. This is quite expected because the average of a randomly sampled set is an unbiased estimation of the average of the entire set. This result indicates that the adjustment term can actually be computed quickly with a small number of additional measurements. The results also show that increasing the dimension of the Euclidean embedding does not help very much; in fact, a lower dimension Euclidean embedding plus the local adjustment terms is sufficient to improve the accuracy of the embedding significantly.

In addition to the improved overall stress, the local adjustment terms also improve the relative errors. As an example, Fig. 16(a) compares the cumulative distribution of the relative errors of the VL-ALL with 7 dimensions (denoted "VL-ALL,7D") with that using the same method with 7 dimensions plus SLAT (denoted as "VL-ALL,7D,SLAT") and with only 2 dimensions plus SLAT (denoted as "VLALL,2D,SLAT") for the King462 data set ${ }^{9}$. The VL-ALL

\footnotetext{
${ }^{9}$ The Euclidean coordinates of the SLAT $(2 \mathrm{D}+1)$ are the first 2 coordinates of the Virtual Landmark 7 dimension embedding.
}

with 2 dimensions plus SLAT attains better performance than that of the pure VL-ALL with 7 dimensions. For example, 90 percentile relative error of "VL-ALL,2D,SLAT" is less than 0.6, but that of "VL-ALL,7D" is larger than 1.0. The performance of "VL-ALL,2D,SLAT' is even better than that of "VL-ALL,7D,SLAT", where 7 dimensions is used. We conclude that adding a (non-Euclidean) local adjustment term is far more effective in improving the accuracy of embedding than adding additional dimensions. More in-depth analysis demonstrates that the performance gain comes largely from improved distance estimation for nodes within the same cluster. However, for the metric, CNL, as can be seen in Fig. 16(b), the performance degrades with SLAT. It means that SLAT option is not good for choosing the closest node. For the metric, RRL, the performance with SLAT is a little better than the one without SLAT as can be seen in Fig. 16(c).

As implied in Theorem 4, a key reason that the hybrid model improves the performance (in relative error) of any Euclidean embedding method lies in the fact that it mitigates its "imperfect estimation" - namely, over-estimates or underestimates - by introducing a (non-Euclidean) local adjustment term that smoothes out (via averaging) the over- and underestimates. This effect can be seen using the skewness metric. Fig. 17 show the resulting skewness measures of the various embedding methods with the SLAT option on, when applied to the King462 data set. Compared with the results in Fig. 9, we see that the fraction near 0 increases considerably. The reduction in skewness is common to all the schemes.

However, it should be noted that if an embedding scheme has large errors but the sum of errors is 0 (not skewed) for all the nodes, LAT cannot improve the performance of the original embedding. For example, in equation (3), the $\tilde{e}_{x}$ can be 0 even if there are errors. Then, there cannot be any improvement from the original embedding scheme.

Our results suggest that due to the existence of prevalent TIVs in the Internet host distance measurement data sets, instead of attempting to develop more sophisticated Euclidean (or metric-based) embedding method that minimizes a global error function (such methods are likely to be more expensive and time-consuming), it is far better to incorporate simpler and less expensive mechanisms to reduce the inevitable (local) estimation errors. Clearly, our proposed hybrid model with localized adjustment terms (LAT) is an example of such a simple but yet effective mechanism. 


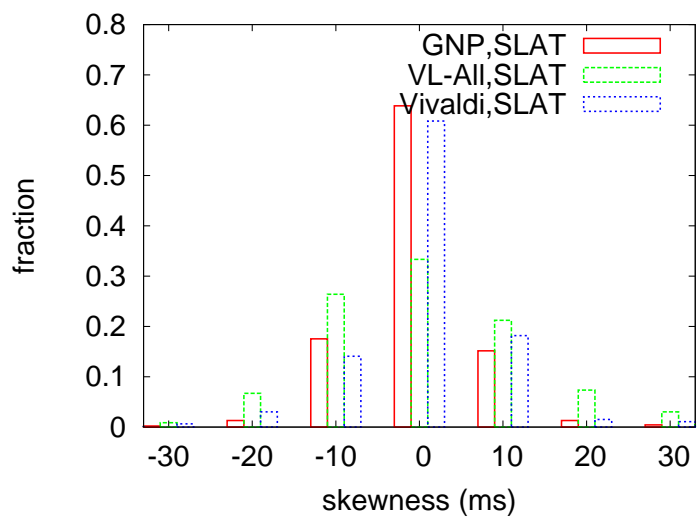

Fig. 17. Cumulative distributions of $s_{x}$ under various schemes with the SLAT option. The data set is King462. The number of dimension is 7.

\section{CONCLUSION}

This paper investigated the suitability of embedding Internet hosts into a Euclidean space given their pairwise distances (as measured by round-trip time). Using the classical scaling and matrix perturbation theories, we established that the (sum of the) magnitude of negative eigenvalues of the (doublycentered, squared) distance matrix as a measure of suitability of Euclidean embedding. Using data sets from real Internet host distance measurements, we illustrated that the distance matrix among Internet hosts contains negative eigenvalues of large magnitude, implying that embedding the Internet hosts in a Euclidean space would incur considerable errors. We attributed the existence of these large-magnitude negative eigenvalues to the prevalence of triangle inequality violations (TIVs) in the data sets. Furthermore, we demonstrated that the TIVs are likely to occur locally, hence the distances among these close-by hosts cannot be estimated very accurately using a global Euclidean embedding. In addition, increasing the dimension of embedding does not reduce the embedding errors.

Based on these insights, we proposed and developed a simple hybrid model that incorporates a localized (non-Euclidean) adjustment term for each node on top of a low-dimensional Euclidean coordinate system. Our hybrid model preserves the advantages of the Euclidean coordinate systems, while improving their efficacy and reducing their overheads (by using a small number of dimensions). Through both mathematical analysis and experimental evaluation, our hybrid model improves the performance of existing embedding methods while using only a low dimension embedding. Lastly, our model can be incorporated into any embedding system (not necessarily Euclidean embedding).

\section{REFERENCES}

[1] S. Lee, Z.-L. Zhang, S. Sahu, and D. Saha, "On suitability of euclidean embedding of internet hosts," in Proc. ACM SIGMETRICS, Saint Malo, France, June 2006.

[2] T. E. Ng and H. Zhang, "Predicting Internet network distance with coordinates-based approaches," in Proc. IEEE INFOCOM, New York, NY, June 2002.

[3] L. Tang and M. Crovella, "Virtual landmarks for the Internet," in Proceedings of the Internet Measurement Conference(IMC), Miami, Florida, Oct. 2003.
[4] H. Lim, J. C. Hou, and C.-H. Choi, "Constructing Internet coordinate system based on delay measurement," in Proceedings of the Internet Measurement Conference(IMC), Miami, Florida, Oct. 2003.

[5] F. Dabek, R. Cox, F. Kaashoek, and R. Morris, "Vivaldi: A decentralized network coordinate system," in Proceedings of ACM SIGCOMM 2004, Portland, OR, Aug. 2004.

[6] M. Costa, M. Castro, A. Rowstron, and P. Key, "Pic: Practical Internet coordinates for distance estimation," in Proceedings of International Conference on Distributed Computing Systems (ICDCS), Tokyo, Japan, Mar. 2004.

[7] H. Zheng, E. K. Lua, M. Pias, and T. G. Griffin, "Internet routing policies and round-trip-times," in The 6th апиual Passive and Active Measurement Workshop, Boston, MA, Mar. 2005.

[8] E. K. Lua, T. Griffin, M. Pias, H. Zheng, and J. Crowcroft, "On the accuracy of embeddings for internet coordinate systems," in Proceedings of the Internet Measurement Conference(IMC), Boston, MA, Apr. 2005.

[9] B. Wong, A. Slivkins, and E. G. Sirer, "Meridian: A lightweight network location service without virtual coordinates," in Proceedings of ACM SIGCOMM 2005, Philadelphia, PA, Aug. 2005.

[10] I. Borg and P. Groenen, Modern Multidimensional Scaling : Theory and Applications. Springer, 1997.

[11] G. H. Gulub and C. F. van Loan, Matrix Computation, 3rd ed. the John Hopkins University Press, 1996.

[12] T. M. Gil, F. Kaashoek, J. Li, R. Morris, and J. Stribling, "King462 data set," http://pdos.lcs.mit.edu/p2psim/kingdata, current year.

[13] "King2305 data set," http://www.cs.cornell.edu/People/egs/meridian/data.php.

[14] J. Stribling, "Rtt among planetlab nodes," http://www.pdos.lcs.mit.edu/ strib/pl_app/, current year.

[15] "Global nework positioning (gnp)," http://www-2.cs.cmu.edu/ eugeneng/research/gnp/.

[16] A. Gupta, "Embedding tree metrics into low-dimensional euclidean spaces," Discrete and Computational Geometry, vol. 24, no. 1, pp. 105116, May 2000.

[17] "Network coordinate research at harvard," http://www.eecs.harvard.edu/s̃yrah/nc/.

[18] A. Y. Ng, M. I. Jordan, and Y. Weiss, "On spectral clustering : Analysis and an algorithm," Advances in Neural Information Processing Systems (NIPS), vol. 14, 2002.

[19] D. B. West, Introduction to Graph Theory, 2nd ed. Prentice Hall., 2001.

\section{APPENDIX}

Proof of Theorem 2. First, verifying whether there exists a maximal TIV-free set of size $k$ among a set of nodes with a distance matrix can be done in polynomial time by enumerating all set of size $k$ and checking the TIVs in a non-deterministic machine. Hence this problem is $N P$.

Now we prove that the problem is NP-hard by reducing the MAXCLIQUE problem (namely, finding the maximal clique in a graph $G$, a well-known NP-complete problem [19]) to the maximal TIV-free set problem. Let $G$ be a connected undirected graph with $n>2$ nodes. We assume that the size of maximal clique of $G$ is $k>2$. (The case $k=2$ is trivial, as any pair of vertices with an edge is a maximal clique.) We construct a distance matrix $D=\left(d_{i j}\right)$ among the set of vertices of $G$ as follows, where $d_{i j}$ will be the defined distance between vertices $i$ and $j$. For each vertex $i$, we set $d_{i i}=0$. For each edge $e_{i j}$ between vertices $i$ and $j$, we set $d_{i j}=1$ and $d_{j i}=1$. Note that for any triangle in $G$, the corresponding distances in $D$ do not violate triangle inequality. For the pair of vertices $i$ and $j$ that do not have an edge between them in $G$, we first set $d_{i j}:=$ undefined. Now, we define all the undefined $d_{i j}$ as follows. For an undefined $d_{i j}$, we compute $c=\max _{k}\left(d_{i k}+d_{k j}\right)$ for all $k$ such that $d_{i k}$ and $d_{k j}$ are already defined. If no such $c$ can be computed because $d_{i k}$ and $d_{k j}$ are undefined for all $k$, we set $c=0$. Then, we set $d_{i j}:=d_{j i}:=c+1$. This transformation takes polynomial time, $O\left(n^{3}\right)$, since there are $n^{2}$ entries in $D$ and for each entry $O(n)$ computation is required.

It can be easily shown that that a triple of nodes $(i, j, k)$ in $G$ forms a triangle if and only if $i, j$, and $k$ do not violate triangle inequality with $d_{i j}, d_{i k}$, and $d_{j k}$ in $D$ (we omit the detailed proof for the sake of space). This means that the maximal TIV-free set with the distances 
defined as $D$ is the maximal clique in $G$. We conclude that finding a maximal TIV-free set problem is NP-hard. Since maximal TIV-free set problem is NP and NP-hard, it is NP-complete.

Proof of Lemma 1. Note that $t_{c}>t_{a}+t_{b}$. Let $\left(\hat{t_{a}}, \hat{t_{b}}, \hat{t_{c}}\right)$ be a metric embedding of the 3 nodes. Then, they should satisfy the triangle inequality constraint as follows.

$$
\hat{t_{a}}+\hat{t_{b}} \geq \hat{t_{c}}, \hat{t_{a}}+\hat{t_{c}} \geq \hat{t_{b}}, \hat{t_{b}}+\hat{t_{c}} \geq \hat{t_{a}}
$$

The squared estimation error, $e$, in this embedding is

$$
e=\left(t_{a}-\hat{t_{a}}\right)^{2}+\left(t_{b}-\hat{t_{b}}\right)^{2}+\left(t_{c}-\hat{t_{c}}\right)^{2}
$$

Let $k:=\left|t_{a}-\hat{t_{a}}\right|+\left|t_{b}-\hat{t_{b}}\right|+\left|t_{c}-\hat{t_{c}}\right|$. We now show that $k \geq\left(t_{c}-t_{a}-t_{b}\right)$. Suppose $k<\left(t_{c}-t_{a}-t_{b}\right)$. There are 8 cases based on the signs of $\left(t_{a}-\hat{t_{a}}\right),\left(t_{b}-\hat{t_{b}}\right)$, and $\left(t_{c}-\hat{t_{c}}\right)$. First, consider the case where $\left(t_{a}>\hat{t_{a}}\right),\left(t_{b}>\hat{t_{b}}\right)$, and $\left(t_{c}>\hat{t_{c}}\right)$. Then,

$$
\begin{aligned}
k & -\left(t_{c}-t_{a}-\hat{t_{b}}\right) \\
& =\left(\hat{t_{a}}-\hat{t_{a}}\right)+\left(\hat{t_{b}}-\hat{t_{b}}\right)+\left(\hat{t_{c}}-\hat{t_{c}}\right)-\left(t_{c}-t_{a}-t_{b}\right) \\
& =2\left(t_{a}+\hat{t_{b}}\right)-\hat{t_{a}}-\hat{t_{b}}-\hat{t_{c}} \\
& >2\left(\hat{t_{a}}+\hat{t_{b}}\right)-\hat{t_{a}}-\hat{t_{b}}-\hat{t_{c}} \\
& >\hat{t_{a}}+\hat{t_{b}}-\hat{t_{c}} \geq 0
\end{aligned}
$$

So $k>\left(t_{c}-t_{a}-t_{b}\right)$, a contradiction. It can be easily shown that all the other 7 cases contradict, too. So we conclude that $k \geq\left(t_{c}-\right.$ $\left.t_{a}-t_{b}\right)$.

Now, for any such $k \geq\left(t_{c}-t_{a}-t_{b}\right)$, consider another embedding $\left(\tilde{t_{a}}, \tilde{t_{b}}, \tilde{t_{c}}\right)$ such that $\tilde{t_{a}}=t_{a}+\frac{k}{3}, \tilde{t_{b}}=t_{b}+\frac{k}{3}$, and $\tilde{t_{c}}=t_{c}-\frac{k}{3}$. Since it can be easily shown that $\left(\tilde{t_{a}}, \tilde{t_{b}}, \tilde{t_{c}}\right)$ satisfies the triangle inequality constraint, it is a metric embedding.

Furthermore, for any such $k \geq\left(t_{c}-t_{a}-t_{b}\right)$, (5) is minimized with this embedding because

$$
\left|t_{a}-\tilde{t_{a}}\right|=\left|t_{b}-\tilde{t_{b}}\right|=\left|t_{c}-\tilde{t_{c}}\right|=\frac{k}{3}
$$

Therefore, $e \geq\left(\frac{k}{3}\right)^{2}+\left(\frac{k}{3}\right)^{2}+\left(\frac{k}{3}\right)^{2}=\frac{k^{2}}{3} \geq \frac{\left(t_{c}-t_{a}-t_{b}\right)^{2}}{3}$.

Proof of Theorem 3 Let $E$ be the sum of squared error of $n$ nodes. $E=\sum_{i}\left(\hat{d}_{i}-d_{i}\right)^{2}$, where $d_{i}$ is a distance between a pair of nodes (called $i$ ) and $\hat{d}_{i}$ is the embedded distance of the pair $i$. There are $n(n-1) / 2$ pairs. Since there are $n(n-1)(n-2) / 6$ triples among $n$ nodes, $E$ can be rewritten by the triples of nodes as follows. $E=\frac{1}{n-2} \sum_{t \in T}\left(\left(\hat{t_{a}}-t_{a}\right)^{2}+\left(\hat{t_{b}}-t_{b}\right)^{2}+\left(\hat{t_{c}}-t_{c}\right)^{2}\right)$, where $T$ is the set of triples and $t_{a}, t_{b}$, and $t_{c}$ are the three distances of a triple $t$, and $\hat{t_{a}}, \hat{t_{b}}$, and $\hat{t_{c}}$ are the corresponding embedded distances. Clearly, $E \geq \frac{1}{n-2} \sum_{t \in V}\left(\left(\hat{t_{a}}-t_{a}\right)^{2}+\left(\hat{t_{b}}-t_{b}\right)^{2}+\left(\hat{t_{c}}-t_{c}\right)^{2}\right)$, where $V \subset T$ is the set of TIV triples. From Lemma 1, we have $E \geq \frac{1}{3(n-2)} \sum_{t \in V}\left(t_{c}-t_{a}-t_{b}\right)^{2}$.

Proof of Theorem 4 We just describe a sketchy of the proof. Let $s_{1}$ be the stress of using the pure Euclidean based scheme. Let $s_{2}$ be the stress of using the pure Euclidean based scheme with the adjustment term.

$$
\begin{aligned}
s_{1}^{2} & =\frac{\sum_{x, y}\left(d_{x y}-d_{x y}^{E}\right)^{2}}{\sum_{x y} d_{x y}^{2}} \\
s_{2}^{2} & =\frac{\sum_{x, y}\left(d_{x y}-d_{x y}^{E}-e_{x}-e_{y}\right)^{2}}{\sum_{x, y} d_{x y}^{2}}
\end{aligned}
$$

$$
\begin{aligned}
& \left(\sum_{x, y} d_{x y}^{2}\right)\left(s_{1}^{2}-s_{2}^{2}\right) \\
= & \sum_{x, y}\left(d_{x y}-d_{x y}^{E}\right)^{2}-\sum_{x, y}\left(d_{x y}-d_{x y}^{E}-e_{x}-e_{y}\right)^{2}
\end{aligned}
$$

Using (2) and reformatting the formula, the final result can be easily obtained.

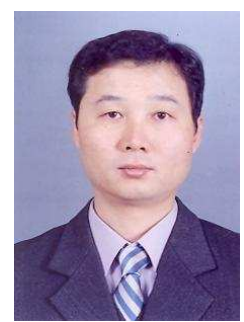

Sanghwan Lee received the BS and MS degree in computer science from Seoul National University, Seoul, Korea, in 1993 and 1995 respectively, and the $\mathrm{Ph} . \mathrm{D}$. degree in computer science and engineering from the University of Minnesota, Minneapolis, in 2005.

Dr. Lee is currently an Assistant Professor at Kookmin University, Seoul, Korea. From June 2005 to February 2006, he worked at IBM T.J. Watson Research Center, Hawthorne, NY. His main research interest is theory and services in the Internet. He is currently applying the embedding schemes to a live peer to peer multimedia streaming system to see the real impact of the accuracy on the application perspective performance.

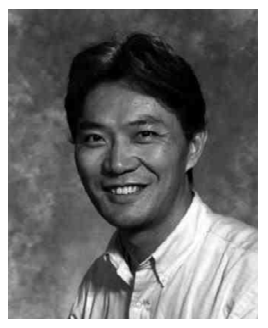

Zhi-Li Zhang received the Ph.D. degree in computer science from the University of Massachusetts, Boston, in 1997.

In 1997, he joined the Computer Science and Engineering Faculty, University of Minnesota, Minneapolis, where he is currently a Full Professor.

Dr. Zhang was a recipient of the National Science Foundation CAREER Award in 1997. He has also been awarded the prestigious McKnight Land-Grant Professorship and George Taylor Distinguished Research Award at the University of Minnesota, and the Miller Visiting Professorship at Miller Institute for Basic Sciences, University of California, Berkeley. He is a co-recipient of an ACM SIGMETRICS Best Paper Award and an IEEE International Conference on Network Protocols (ICNP) Best Paper Award.

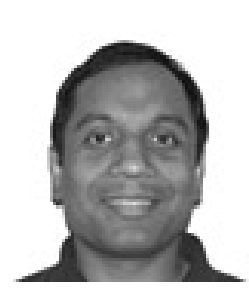

Sambit Sahu is a research staff member in the Networking Software and Services Group. He received a Ph.D. degree in computer science from the University of Massachusetts at Amherst in 2001. He joined IBM in 2001. His research has focused on overlay-based communication, network configuration management, content distribution architecture, and design and analysis of high-performance network protocols. Dr. Sahu has published a number of papers in the areas of differentiated services, multimedia, overlay-based communication, and network management and has over 20 patents filed in these areas.

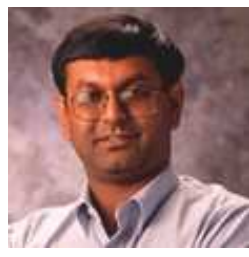

Debanjan Saha is with the Security, Privacy, and Networking Department at IBM Research. He leads a group of researchers working on different aspects of network software and services. He received a B.Tech. degree in computer science and engineering from the Indian Institute of Technology in 1990, and M.S. and Ph.D. degrees in computer science from the University of Maryland at College Park in 1992 and 1995, respectively. Dr. Saha has authored more than 50 papers and standards contributions and is a frequent speaker at academic and industry events. He serves regularly on the executive and technical committees of major networking conferences.
Since the denominators are the same, we compute $\left(\sum_{x, y} d_{x y}^{2}\right)\left(s_{1}^{2}-s_{2}^{2}\right)$ to compute $s_{1}^{2}-s_{2}^{2}$. 